\title{
Channel Acquisition for Wideband CDMA Signals
}

\author{
Vinayak Tripathi, Student Member, IEEE, Ashok Mantravadi, Student Member, IEEE, and \\ Venugopal V. Veeravalli, Senior Member, IEEE
}

\begin{abstract}
The problem of initial acquisition of the channel parameters of a wideband CDMA signal received in a multipath fading environment with multiaccess interference is considered. Since the signal is wideband, the fading is frequency selective and the parameters of interest are the (complex) gains and delays in the corresponding tapped delay line model for the channel. The scenario considered is one where a single new user is to be acquired on the reverse link by the base station, and where the channel parameters of the interfering users are known. Following a minimum mean squared error (MMSE) strategy for suppressing the multiaccess interference, the parameter estimation problem is posed in a maximum likelihood framework. To reduce complexity, the solution is implemented in two stages: first, the estimated tap delays are restricted to be at chip spacings; second, the number of taps is reduced by allowing for arbitrary spacing between them. The performance of the proposed techniques is studied through numerical simulations. It is shown that significant gains can be obtained by exploiting the structure of the interference and acquiring the channel parameters jointly.
\end{abstract}

Index Terms-Bandlimited communication, code division multiaccess, fading channels, least mean square methods, maximum likelihood estimation, multiuser channels.

\section{INTRODUCTION}

$\mathbf{C}^{2}$ ODE DIVISION multiple access (CDMA) signals, particularly wideband CDMA signals, are likely to have bandwidths that are considerably larger than the inverse of the delay spread of the multipath propagation environment. Hence, CDMA signals typically undergo frequency selective fading, and the channel appears as a tapped delay line to the signals. Accurate estimates of the channel parameters (i.e., tap delays and complex gains) are needed prior to diversity combining and symbol detection. The process of obtaining initial estimates of these parameters is called acquisition.

The acquisition problem is relatively easy on the forward link of a cellular communication system for two reasons: 1) there is usually a pilot signal that assists channel estimation; and 2) the signals of all the in-cell interferers see the same channel that the intended signal sees. Our focus is hence on the reverse link of a CDMA system, where the received signal at the base station consists of the sum of the asynchronous signals of the users,

Manuscript received August 1999; revised February 28, 2000. This work was supported by the NSF under Grant NCR-9523967 and the CAREER/PECASE Grant CCR-9733204. This work was presented in part at the IEEE Vehicular Technology Conference, Tokyo, Japan, May 2000.

V. Tripathi and A. Mantravadi are with the School of Electrical Engineering, Cornell University, Ithaca, NY 14853 USA (e-mail: tripathi@ee.cornell.edu; ashok@ee.cornell.edu).

V. V. Veeravalli was with Cornell University. He is now with the ECE Department and the Coordinated Science Laboratory, University of Illinois at Urbana-Champaign, IL 61801 USA (e-mail: vvv@uiuc.edu).

Publisher Item Identifier S 0733-8716(00)06115-1. each of which sees a different multipath channel. The acquisition of a new user by the base station is hence severely affected by multiaccess interference (MAI). Our goal is to design acquisition schemes that are resistant to MAI.

The conventional approach to multitap channel acquisition, that is used in IS-95 based CDMA systems [19, p. 84], involves acquiring one tap of the desired signal (usually the strongest), and then searching for additional taps around the acquired tap. In addition, the conventional acquisition scheme treats the MAI as additive random noise without exploiting any of its structure. In recent work, Rick and Milstein [10] considered the joint acquisition of the taps for a single user in additive white Gaussian noise. Clearly, acquisition can be further improved if the MAI can be cancelled or suppressed. There have been several papers on acquisition schemes that are resistant to MAI, some of which are applicable to multitap channel acquisition. These include subspace-based decomposition techniques (e.g., [12]), maximumlikelihood techniques involving sample statistics [14], [13], and joint MMSE acquisition and detection [3], [4]. The underlying assumption in all of this work is that the spreading sequences of the users repeat every symbol period (short sequences). But short sequences may be impractical for the asynchronous reverse link. Short sequences allow for the possibility that two (or more) users have signals that are highly correlated over several bit intervals, reducing the worst case performance and reuse efficiency [18], [17], [8].

Practical wireless CDMA systems, such as those specified in the IS-95 standard [16] and the CDMA 2000 proposal [15], randomize the users' signals on the reverse link by using spreading sequences whose periods are much greater than the spreading factor. It is of interest to develop acquisition schemes that are applicable to such systems and are resistant to MAI. In this work, we extend the single-path MAI resistant acquisition proposed in [7], to the joint acquisition of the taps of a frequency selective fading channel. Although our scheme is derived for systems that use long spreading sequences, it can be applied to those that use short spreading sequences as well.

We consider the situation where the channel parameters of a single new user need to be estimated. If more than one user enters the system at the same time, we can assume that the users are acquired one at a time. Hence, since the existing users in the system have already been acquired and are being successfully demodulated, it is reasonable to assume that their channel parameters are known. In such a situation, it is possible to acquire the new user by using an interference cancellation approach, where the interfering signals are reconstructed and subtracted from the received signal (see, e.g., [1]). However, such a scheme would have to use code-symbol estimates, which may be unreliable for low rate error control coding and matched-filter detection that is typically used in conjunction with long sequences. Alternatively, the decoded symbols would have to be used to 
reconstruct the code symbols, which may be impractical due to the complexity and decoder delay involved. Consequently, we do not assume knowledge of the code symbols of the interfering users. Instead, we implicitly use soft estimates based on a minimum mean squared error (MMSE) criterion, and pose the channel estimation problem in a maximum-likelihood (ML) framework based on the resultant signal. It is important to note that in random sequence CDMA systems, MMSE detection of the data symbols may be impractical, since the detector needs to be updated at the symbol rate. However, using MMSE symbol estimates for acquisition with a sufficiently long preamble does not have the same timing constraints and should be practical.

The frequency selective fading channel may be modeled as a tapped delay line with equally spaced taps where the number of taps is determined by the delay spread of the channel [9, p. 795]. However, this model may lead to an unnecessarily large number of taps if only a subset of the taps capture significant signal energy. Hence, we consider ways to reduce the number of taps (and hence, the complexity of postacquisition processing at the receiver) while retaining most of the signal energy. In order to improve the approximation with the smaller number of taps, we allow for arbitrary spacing between them. We consider an optimal approach to reducing the number of taps based on maximizing a correlation performance metric, as well as two suboptimal approaches that are easier to implement.

The paper is organized as follows. In Section II, we briefly review the frequency selective channel model, and apply it to an asynchronous CDMA system. In Section III, we derive the ML acquisition scheme under the framework outlined above. We also develop schemes to reduce the number of taps. In Section IV, we study the performance of the various joint tap acquisition schemes, and also compare the performance with that of a simple tap-by-tap acquisition scheme. Finally, in Section V, we give our conclusions and outline topics for future research on this problem.

\section{SySTEM MODEL}

Consider a band-limited signal $s(t)$ with complex baseband bandwidth of $W / 2$ (i.e., a passband bandwidth of $W$ ) transmitted through a multipath propagation channel. The channel is well modeled as a linear time varying system [9], with impulse response that we denote by $h(t, \xi)$. The received signal can then be written as

$$
r(t)=\int_{-\infty}^{\infty} h(t, \xi) s(t-\xi) d \xi .
$$

Without loss of generality, we can assume that the channel is of bandwidth $W / 2$ as well, ${ }^{1}$ i.e.,

$$
H(t, f):=\int_{-\infty}^{\infty} h(t, \xi) e^{-j 2 \pi f \xi} d \xi \approx 0 \quad \text { for }|f|>W / 2 .
$$

Hence, applying the sampling theorem to $h(t, \xi)$, we have

$$
h(t, \xi)=\sum_{\ell=-\infty}^{\infty} h(t, \ell / W) \operatorname{sinc}(W \xi-\ell) .
$$

${ }^{1}$ Of course, the time variations in the channel will result in a Doppler spreading of the bandwidth; we are assuming that $W / 2$ includes guard bands that account for this Doppler spread.
Plugging (2) into (1) we get

$$
\begin{aligned}
r(t) & =\sum_{\ell=-\infty}^{\infty} h(t, \ell / W) \int_{-\infty}^{\infty} s(t-\xi) \operatorname{sinc}(W \xi-\ell) d \xi \\
& =\frac{1}{W} \sum_{\ell} h(t, \ell / W) s(t-\ell / W) .
\end{aligned}
$$

Thus, the channel can be represented by a set of discrete taps of strength $h(t, \ell / W)$ spaced at intervals of $1 / W$; this is the tapped delay line channel model. Note that the representation is independent of the origin of the $\xi$ axis. In particular, (3) is valid even when we have flat fading for which the channel can be represented by a single path with time varying gain and delay, i.e.,

$$
\begin{aligned}
h(t, \xi) & =\left[h_{0}(t) \delta\left(\xi-\xi_{0}(t)\right)\right] * \operatorname{sinc}(W \xi) \\
& =h_{0}(t) \operatorname{sinc}\left(W\left(\xi-\xi_{0}(t)\right)\right.
\end{aligned}
$$

and $\xi_{0}(t)$ is not a multiple of $1 / W$. Here, * represents the convolution operation, and convolving with $\operatorname{sinc}(W \xi)$ in (4) bandlimits the channel to $W / 2$. Hence, although the channel is really a single tap channel with arbitrary time-varying delay $\xi_{0}(t)$, it can be represented by a tapped delay line with taps spaced at fixed delays $\{\ell / W\}$. However, to get a reasonable approximation with the tapped delay line, we would need multiple taps.

For the representation (3) to be valid in general, we must allow for an infinite number of taps. However, for a frequency selective fading channel with delay spread $\tau_{\mathrm{DS}}, h(t, \ell / W)$ is significant only for a finite number, $L_{c}$, of the taps. Since the taps are restricted to be at $1 / W$ spacing with respect to the origin on $\xi$ axis, $L_{c}$ may in general be greater than $\left\lceil\tau_{\mathrm{DS}} W\right\rceil$ (as in the flat fading case noted above). Assume that the first significant tap is at delay $d(t)$, where $d(t)$ is some multiple of $1 / W$ and could possibly vary with time. The band-limited channel response can then be written as

$$
\begin{aligned}
h(t, \xi) & =\left(\sum_{\ell=d(t)}^{d(t)+L_{c}-1} h(t, \ell / W) \delta(\xi-\ell / W)\right) * \operatorname{sinc}(W \xi) \\
& =\sum_{\ell=d(t)}^{d(t)+L_{c}-1} h(t, \ell / W) \operatorname{sinc}(W \xi-\ell) \\
& =\sum_{\ell=0}^{L_{c}-1} h(t,(\ell+d(t)) / W) \operatorname{sinc}(W \xi-\ell-d(t))
\end{aligned}
$$

In CDMA systems that use chip waveforms that approximate Nyquist sinc pulses, the chip period $T_{c} \approx 1 / W$, and the above approach allows us to consider a chip-synchronous model even when the users are asynchronous. However, the number of taps in such a model may be prohibitively large, and we will be interested in reducing the number of taps by allowing for arbitrary spacing between the taps. Clearly, for a channel model consisting of $L$ taps with time-varying complex 
gains $h_{1}(t), \ldots, h_{L}(t)$ at delays $\tau_{1}(t) / W, \ldots, \tau_{L}(t) / W$, the band-limited channel response is

$$
\begin{aligned}
h(t, \xi) & =\left(\sum_{m=1}^{L} h_{m}(t) \delta\left(\xi-\tau_{m}(t) / W\right)\right) * \operatorname{sinc}(W \xi) \\
& =\sum_{m=1}^{L} h_{m}(t) \operatorname{sinc}\left(W \xi-\tau_{m}(t)\right) .
\end{aligned}
$$

For the remainder of the paper, we consider the channel to be invariant over the observation window for the acquisition stage, i.e., the fading is assumed to be slow. We hence drop the time variable $t$ in denoting the channel response.

We now apply the channel model discussed above to the asynchronous (reverse link) CDMA system with $K$ users and linear modulation. The received complex baseband ${ }^{2}$ signal is given by

$$
\begin{gathered}
r(t)=\sum_{k=1}^{K} \sum_{\ell=0}^{L_{c, k}-1} \sum_{n=0}^{M^{\prime}-1} \beta_{k, \ell} \sqrt{\mathcal{E}_{k}} b_{k, n} c_{k}^{(n)}\left(t-\left(d_{k}+\ell\right) / W\right) \\
+w(t), \quad t \in\left[0, M T_{s}\right]
\end{gathered}
$$

where

$b_{k, n} \quad$ is symbol $n$ of user $k$, and $T_{s}$ is the symbol period; $\mathcal{E}_{k} \quad$ is the average received symbol energy summed over all the paths of user $k$; and

$c_{k}^{(n)}(t) \quad$ is given by

$$
c_{k}^{(n)}(t)=\sum_{j=0}^{N-1} c_{k, j}^{(n)} \psi\left(t-n T_{s}-j T_{c}\right)
$$

where $T_{c}$ is the chip period, and the ratio $N=$ $T_{s} / T_{c}$ is the spreading factor of the system.

The sequence $c_{k, j}^{(n)}$ is the (complex) chip sequence of the $k$ th user. If $c_{k, j}^{(n)}=c_{k, j}^{(0)}$ for all $j$ and $n$, the sequence is said to be short. If $c_{k, j}^{(n)}$ does not exhibit this periodicity, then it is said to long or random.

The chip waveform $\psi(t)$ has unit energy, and the spreading waveform corresponding to each symbol is also normalized to have unit energy by normalizing the corresponding chip sequence. We assume that $\psi(t)$ is chosen to be a good approximation to the Nyquist sinc pulse, i.e., $\psi(t) \approx \sqrt{W} \operatorname{sinc}(W t-0.5)$. In the numerical results, we model $\psi(t)$ as a sinc pulse truncated to a length of 9 chips.

- $L_{c, k}$ is the number of taps of the $k$ th user channel. Note that on the reverse link the users see different propagation channels.

- $\beta_{k, \ell}$ is the channel gain at tap $\ell$ of user $k$, and the tap gains are, in general, modeled as random variables with variances normalized so that

$$
\sum_{\ell=1}^{L_{c, k}} E\left|\beta_{k, \ell}\right|^{2}=1 \text {. }
$$

- $d_{k}$ is the offset due to propagation delay of user $k$, which can be assumed to be an integer without loss of generality

${ }^{2}$ If $y_{p}(t)$ is a real passband signal with carrier frequency $f_{c}$, and $y_{b}(t)$ is the corresponding baseband complex envelope, we assume that $y_{p}(t)=\operatorname{Re}\left[\sqrt{2} y_{b}(t) e^{j 2 \pi f_{c} t}\right]$. (based on the arguments given in the beginning of this section).

- $w(t)$ is a zero mean circularly complex Gaussian (CCG) process with autocorrelation function

$$
R_{w}(\tau)=E\left[w^{*}(t) w(t+\tau)\right]=N_{0} \delta(\tau)
$$

Note that $w(t)$ may be assumed to include out-of-cell interference as well.

We consider an observation interval that encompasses $M$ symbol intervals. If the users are synchronous, the fading is flat, and the chip waveform $\psi(t)$ is limited to $T_{c}$, then exactly $M$ symbols of each user contribute to the observation interval. However, since the users are asynchronous, the fading is frequency selective, and we consider chip waveforms that span several chips, more than $M$ symbols could contribute. The number of symbols that contribute significantly to the observation interval is denoted by $M^{\prime}$.

The new user entering the system is assumed to be the one corresponding to $k=1$. In addition, we assume the following about the system.

- The initial timing uncertainty is $J$ chips, i.e., $d_{1} \in\{1,2, \ldots, J\}$.

- The new user transmits a preamble with known symbols over the observation interval. Without loss of generality, we can assume that $b_{1, n}=1$, for $n=0, \ldots, M^{\prime}-1$.

- The channel responses of the interfering users are known since they are already being demodulated. However, the (code) symbols transmitted by the interfering users are not known.

Under the above assumptions, the received signal of interest can be expressed as

$$
\begin{gathered}
r(t)=\sum_{\ell=0}^{L_{c}-1} a_{1, \ell} c_{1}\left(t-\left(d_{1}+\ell\right) T_{c}\right)+\sum_{n=0}^{M^{\prime}-1} \sum_{k=2}^{K} \sum_{\ell=0}^{L_{c, k}-1} \\
\cdot a_{k, \ell} b_{k, n} c_{k}^{(n)}\left(t-\left(d_{k}+\ell\right) T_{c}\right)+w(t)
\end{gathered}
$$

where $a_{k, \ell}=\beta_{k, \ell} \sqrt{\mathcal{E}_{k}}$, and $c_{1}(t)=\sum_{n=0}^{M^{\prime}-1} c_{1}^{(n)}(t)$. Note that for convenience we have dropped the subscript " 1 " in $L_{c, 1}$.

Since user 1 employs a preamble, this model can be converted to a problem involving a maximum of $K_{\text {eff }}=M^{\prime}(K-1)+1$ effective users by separating the signals corresponding to each symbol of each interfering user in the observation interval. Define

$$
\begin{aligned}
\tilde{c}_{1, j}(t) & =c_{1}\left(t-d_{1} T_{c}-(j-1) T_{c}\right) \\
\tilde{c}_{M^{\prime}(k-2)+n+2, j}(t) & =c_{k}^{(n)}\left(t-d_{k} T_{c}-(j-1) T_{c}\right) \\
& \text { for } n=0, \ldots, M^{\prime}-1
\end{aligned}
$$

where $k=2, \ldots, K$ is the interfering user index, $j=1, \ldots, L_{c, k}$ is the path index, and $t \in\left[0, M T_{s}\right]$. Consequently, (8) can be rewritten as

$$
r(t)=\sum_{j=1}^{L_{c}} a_{1, j} \tilde{c}_{1, j}(t)+\sum_{k=2}^{K_{\mathrm{cff}}} b_{k}^{\prime} \sum_{j=1}^{L_{c, k}} a_{k, j}^{\prime} \tilde{c}_{k, j}(t)+w(t),
$$$$
t \in[0, T]
$$ 
where, $T=M T_{s}$, and for $n=0, \ldots, M^{\prime}-1$, and $k=$ $2, \ldots, K$

$$
a_{M^{\prime}(k-2)+n+2, \ell}^{\prime}=a_{k, \ell}
$$

and

$$
b_{M^{\prime}(k-2)+n+2}^{\prime}=b_{k, n} .
$$

The first step of the proposed channel estimation algorithm involves generating discrete statistics from the observed continuous signal. One way to produce these statistics is through chip matched filtering.

Remark 1: Statistics generated by chip matched filtering, where the received continuous-time signal is projected onto shifted versions of the chip pulse function $\psi\left(t-i T_{c}\right), i=0, \ldots, M N-1$, are approximately sufficient as long as the shifted chip waveforms are roughly orthogonal and $T_{c} \approx 1 / W$, i.e., $\psi(t) \approx \sqrt{W} \operatorname{sinc}(W t-0.5)$ [6].

Chip matched filtering produces a vector $y$ of dimension $D=$ $M N$ with

$$
\begin{aligned}
y_{n} & =\left\langle r(t), \psi\left(t-i T_{c}\right)\right\rangle_{T} \\
& =\int_{0}^{T} r(t) \psi\left(t-i T_{c}\right) d t \quad \text { for } i=1, \ldots, D
\end{aligned}
$$

where ${ }^{3}$

$$
\langle f, g\rangle_{T}=\int_{0}^{T} f(t) g^{*}(t) d t
$$

is the inner product over the observation interval $[0, T]$. Since this is a linear operation, the resulting vector $\boldsymbol{y}=\left[\begin{array}{llll}y_{1} & y_{2} & \ldots & y_{D}\end{array}\right]^{\top}$ can be written as a sum of the contributions of each effective user as

$$
\boldsymbol{y}=\sum_{j=1}^{L_{c}} a_{1, j} \boldsymbol{u}_{1, j}+\sum_{k=2}^{K_{c \mathrm{cff}}} b_{k}^{\prime} \sum_{j=1}^{L_{c, k}} a_{k, j}^{\prime} \boldsymbol{u}_{k, j}+\boldsymbol{w}
$$

where the $i$ th component of the vector $\boldsymbol{u}_{k, j}$ is given by

$$
\boldsymbol{u}_{k, j}(i)=\left\langle\psi\left(t-i T_{c}\right), \tilde{c}_{k, j}(t)\right\rangle_{T}
$$

and $w$ is a complex circularly Gaussian (CCG) vector with covariance matrix

$$
\boldsymbol{R}_{\boldsymbol{w}}=E\left(\boldsymbol{w} \boldsymbol{w}^{\dagger}\right)=N_{0} \boldsymbol{I}_{M N}
$$

with $^{\dagger}$ denoting the Hermitian operation.

Since the spreading sequences and channel parameters of the interfering users are assumed to be known, the second summation for the effective users in (13) can be collapsed into a single vector

$$
\boldsymbol{u}_{k}=\sum_{j=1}^{L_{c, k}} a_{k, j}^{\prime} \boldsymbol{u}_{k, j}, \quad k=2, \ldots, K_{\mathrm{eff}} .
$$

Hence, the received vector can be represented compactly using the matrix-vector equation

$$
\boldsymbol{y}=\boldsymbol{U}_{1} \boldsymbol{a}_{1}+\boldsymbol{U b}+\boldsymbol{w}
$$

where $\boldsymbol{U}_{1}=\boldsymbol{U}_{1}\left(d_{1}\right)=\left[\begin{array}{lll}\boldsymbol{u}_{1,1} & \ldots & \boldsymbol{u}_{1, L_{c}}\end{array}\right]$ is the matrix of spreading sequences corresponding to the taps of user 1 , and

${ }^{3}$ For large $M$, the edge effects resulting from limiting the integral to $[0, T]$ become negligible. $\boldsymbol{a}_{1}=\left[\begin{array}{llll}a_{1,0} & \ldots & a_{1, L_{c}-1}\end{array}\right]^{\top}$ is the vector of corresponding tap amplitudes. Furthermore, $\boldsymbol{U}=\left[\begin{array}{lll}u_{2} & \ldots & \boldsymbol{u}_{K_{\text {cff }}}\end{array}\right]$ is the matrix of spreading vectors (combined over the taps) of the interferers, and $\boldsymbol{b}=\left[\begin{array}{llll}b_{2}^{\prime} & \ldots & b_{K_{\mathrm{cff}}}^{\prime}\end{array}\right]^{\top}$ is the vector of their code symbols.

Before proceeding further, we note that a channel estimation algorithm based on $\boldsymbol{y}$ would have to process the matrix $\boldsymbol{U}$, which can be up to a size of $M N \times M^{\prime}(K-1)$. We could trade off the resulting complexity with performance by considering only $K_{c}$ of the $K-1$ interferers, and including the remaining interferers in the noise vector $\boldsymbol{w}$. The variance of the noise components can then be modeled as (see also [6]):

$$
\sigma_{I}^{2}=N_{0}+\sum_{k=K_{c}+2}^{K} \frac{\mathcal{E}_{k}}{N} \sigma_{\psi}^{2}
$$

where $\sigma_{\psi}^{2}=\int_{-\infty}^{\infty} R_{\psi}^{2}(\alpha) d \alpha$, with $R_{\psi}(\alpha)$ being the autocorrelation of the chip waveform, i.e.,

$$
R_{\psi}(\alpha)=\int_{-\infty}^{\infty} \psi(t) \psi\left(t-\alpha T_{c}\right) d t .
$$

As mentioned before, $\boldsymbol{w}$ could include out-of-cell interference as well.

\section{Channel Estimation Algorithms}

Assuming an $L_{c}$ tapped channel model with equally spaced taps, the channel parameters to be estimated are the initial tap delay $d_{1}$ and the gain vector $\boldsymbol{a}_{1}$. This problem is considered first. As mentioned in Section I, we would also like to reduce complexity by reducing the number of taps once the $L_{c}$ tap channel is estimated. Schemes for achieving this reduction are also discussed in this section.

\section{A. MMSE-ML Estimation}

Consider the discrete system model given in (14). Since the new user is still to be acquired, it may not be reasonable or practical to impose a prior distribution on the parameters $d_{1}$ and $\boldsymbol{a}_{1}$. Hence, we adopt the maximum-likelihood criterion for the estimation problem. Also, since we are interested only in $d_{1}$ and $\boldsymbol{a}_{1}$, the interferers' symbols $\boldsymbol{b}$ are essentially (discrete) nuisance parameters. If the distribution of these symbols was known, the likelihood function for the estimation problem would be

$$
p\left(\boldsymbol{y} \mid d_{1}, \boldsymbol{a}_{1}\right)=\sum_{\boldsymbol{b}} p\left(\boldsymbol{y} \mid d_{1}, \boldsymbol{a}_{1}, \boldsymbol{b}\right) p(\boldsymbol{b}) .
$$

Note that, since the noise is assumed to be circularly complex Gaussian (CCG), the conditional probability density function (pdf) $p\left(\boldsymbol{y} \mid d_{1}, \boldsymbol{a}_{1}, \boldsymbol{b}\right)$ is that of CCG vector with mean $\boldsymbol{U}_{1} \boldsymbol{a}_{1}+\boldsymbol{U} \boldsymbol{b}$ and covariance matrix $\sigma_{I}^{2} \boldsymbol{I}_{M N}$. For a coded CDMA system, $p(\boldsymbol{b})$ may in general be difficult to characterize. Moreover, even if we assume that the symbols in $\boldsymbol{b}$ are independent, identically distributed (i.i.d.) taking on equally likely values, it may not be possible to obtain a closed form expression for the likelihood function in (15). Consequently, this approach does not appear promising. However, for most coding schemes in practice, it is the case that the code symbol vector $\boldsymbol{b}$ satisfies $E[\boldsymbol{b}]=$ $0, E\left[\boldsymbol{b}^{\dagger}\right]=\boldsymbol{I}$, as long as information symbols are independent and equally likely. This information about the statistics of $\boldsymbol{b}$ can be exploited using an MMSE approach, as described below. 
For fixed $d_{1}$ and $\boldsymbol{a}_{1}$, we produce an MMSE estimate for the vector $\boldsymbol{b}$ from the effective received vector for the interferers, defined as

$$
z=\boldsymbol{y}-\boldsymbol{U}_{1} \boldsymbol{a}_{1}=\boldsymbol{U} \boldsymbol{b}+\boldsymbol{w} .
$$

The MMSE solution corresponds to $\hat{\boldsymbol{b}}_{\mathrm{MMSE}}=\hat{\boldsymbol{G}} z$, where

$$
\hat{\boldsymbol{G}}=\arg \min _{\boldsymbol{G}} E\left[\|G \boldsymbol{z}-\boldsymbol{b}\|^{2}\right] .
$$

This is a standard problem in estimation theory [2, ch. 12] and has been applied to the CDMA detection problem in the past [5]. The MMSE solution can be given in two alternative forms [2]:

$$
\begin{aligned}
\hat{\boldsymbol{b}}_{\mathrm{MMSE}} & =\left[\boldsymbol{U}^{\dagger}\left(\boldsymbol{U} \boldsymbol{U}^{\dagger}+\sigma_{I}^{2} \boldsymbol{I}\right)^{-1}\right] z \\
& =\left[\left(\boldsymbol{U}^{\dagger} \boldsymbol{U}+\sigma_{I}^{2} \boldsymbol{I}\right)^{-1} \boldsymbol{U}^{\dagger}\right] z .
\end{aligned}
$$

Using $\hat{\boldsymbol{b}}_{\mathrm{MMSE}}$, the maximum-likelihood channel estimation problem for user 1 can be posed as

$$
\left[\hat{d}_{1}, \hat{\boldsymbol{a}}_{1}\right]=\arg \max _{d_{1}, \boldsymbol{a}_{1}} p\left(\boldsymbol{y} \mid d_{1}, \boldsymbol{a}_{1}, \hat{\boldsymbol{b}}_{\mathrm{MMSE}}\right) .
$$

Using the fact that $\boldsymbol{w}$ is CCG, (17) can easily be written as

$$
\begin{aligned}
{\left[\hat{d}_{1}, \hat{\boldsymbol{a}}_{1}\right] } & =\arg \min _{d_{1}, \boldsymbol{a}_{1}}(z-\boldsymbol{U} \hat{\boldsymbol{G}} \boldsymbol{z})^{\dagger}(\boldsymbol{z}-\boldsymbol{U} \hat{\boldsymbol{G}} \boldsymbol{z}) \\
& =\arg \min _{d_{1}, \boldsymbol{a}_{1}}\left(\boldsymbol{y}-\boldsymbol{U}_{1} \boldsymbol{a}_{1}\right)^{\dagger} \boldsymbol{B}\left(\boldsymbol{y}-U_{1} \boldsymbol{a}_{1}\right) \\
& =\arg \max _{d_{1}, \boldsymbol{a}_{1}} \boldsymbol{y}^{\dagger} \boldsymbol{B} \boldsymbol{U}_{1} \boldsymbol{a}_{1}+\boldsymbol{a}_{1}^{\dagger} \boldsymbol{U}_{1}^{\dagger} \boldsymbol{B} \boldsymbol{y}-\boldsymbol{a}_{1}^{\dagger} \boldsymbol{U}_{1}^{\dagger} \boldsymbol{B} \boldsymbol{U}_{1} \boldsymbol{a}_{1}
\end{aligned}
$$

where $\boldsymbol{B}=(\boldsymbol{I}-\boldsymbol{U} \hat{\boldsymbol{G}})^{\dagger}(\boldsymbol{I}-\boldsymbol{U} \hat{\boldsymbol{G}})$, and $\hat{\boldsymbol{G}}$ can be obtained from (16). Since the metric in (18) is a quadratic in $\boldsymbol{a}_{1}$, the estimate of $\boldsymbol{a}_{1}$ as a function of $d_{1}$ is given by

$$
\hat{\boldsymbol{a}}_{1}=\left(\boldsymbol{U}_{1}^{\dagger} \boldsymbol{B} \boldsymbol{U}_{1}\right)^{-1}\left(\boldsymbol{U}_{1}^{\dagger} \boldsymbol{B} \boldsymbol{y}\right) \text {. }
$$

We then substitute (19) back into the likelihood function to obtain an estimate for the delay of the first tap as

$$
\hat{d}_{1}=\arg \max _{d_{1}}\left(\boldsymbol{y}^{\dagger} \boldsymbol{B} \boldsymbol{U}_{1}\right)\left(\boldsymbol{U}_{1}^{\dagger} \boldsymbol{B} \boldsymbol{U}_{1}\right)^{-1}\left(\boldsymbol{U}_{1}^{\dagger} \boldsymbol{B} \boldsymbol{y}\right) .
$$

Note that although we are computing the ML estimate of an $L_{c}$ tap channel, the maximization of the likelihood function in (17) is only over a single variable $d_{1}$. In addition, since $d_{1}$ is an integer, we only need to search for the maximum at $J$ locations, and the matrix $U_{1}$ can be formed directly using shifts of the spreading sequence corresponding to user 1 . In effect, we have a single variable hypothesis testing problem: the $L_{c}$ tap channel response of user 1 can be estimated from (20) by maximizing over the $J$ possible values of $d_{1}$, and then computing the corresponding estimate of $\boldsymbol{a}_{1}$ using (19). It is worth noting that when $K_{c}=0$ and $L_{c}=1$, the MMSE-ML scheme proposed above is the same as the matched filter based single tap acquisition scheme used in IS-95 systems [16]. We refer to this special case as "conventional" acquisition and compare it to the more general case above in Section IV.

The crux of the computation to implement the above MMSE-ML algorithm is taken up in forming the matrix $B$. The matrix inverse involved in the computation has size $M^{\prime} K_{c} \times M^{\prime} K_{c}$, and hence its computation would require $O\left(M^{3}\right)$ operations. As we increase the preamble size to improve performance, this computation may prove too cumbersome. An alternate approach in this case would be to process the longer preamble in short blocks and combine the results [7]. Specifically, assume we have an observation of length $b M$ symbols

$$
\boldsymbol{y}=\left[\begin{array}{lll}
\boldsymbol{y}_{1}^{\top} & \ldots & \boldsymbol{y}_{b}^{\top}
\end{array}\right]^{\top}
$$

where $\boldsymbol{y}_{i}, i=1, \ldots, b$, are of length $M N$. We could then pose an ML problem in terms of the vectors $\boldsymbol{B}_{1} \boldsymbol{y}_{1}, \ldots, \boldsymbol{B}_{b} \boldsymbol{y}_{b}$, where $B_{i}$ is the matrix $\boldsymbol{B}$ for the $i$ th block of observations. The corresponding solution can easily be shown to be

$$
\begin{aligned}
& \hat{\boldsymbol{a}}_{1}=\boldsymbol{P}^{-1} \boldsymbol{v} \\
& \hat{d}_{1}=\arg \max _{d_{1}} \boldsymbol{v}^{\dagger} \boldsymbol{P}^{-1} \boldsymbol{v}
\end{aligned}
$$

where

$$
\begin{aligned}
\boldsymbol{v} & =\sum_{i=1}^{b} \boldsymbol{U}_{1, i}^{\dagger} \boldsymbol{B}_{i} \boldsymbol{y}_{i} \\
\boldsymbol{P} & =\sum_{i=1}^{b} \boldsymbol{U}_{1, i}^{\dagger} \boldsymbol{B}_{i} \boldsymbol{U}_{1, i}
\end{aligned}
$$

with $\boldsymbol{U}_{1, i}\left(d_{1}\right)$ being the spreading sequence matrix of user 1 over the $i$ th block. Note that the above block processing would be simpler when short sequences are used, since the matrix $\boldsymbol{B}_{i}$ would be independent of $i$ and would have to be computed only once.

\section{B. Reduction of Taps}

In the second stage of the channel estimation algorithm, we are interested in reducing the number of taps in the channel response to $L_{r}<L_{c}$ while retaining most of the signal energy. The main motivation behind this stage is to reduce the complexity of the receiver. In a channel having a large delay spread, it is possible that most of the signal energy is concentrated in a few distinct pockets. ${ }^{4}$ Hence, although $L_{c}$ is quite large, only a subset of the taps may receive significant energy. In such a situation, the complexity of the receiver can be reduced by employing fewer taps for the channel response. Referring back to (5), we can define the actual band-limited channel response function in terms of the tap amplitudes $\boldsymbol{a}_{1}$ and the delay $d_{1}$ as

$$
h_{c}(\xi)=\sum_{\ell=0}^{L_{c}-1} a_{1, \ell} \operatorname{sinc}\left(W \xi-d_{1}-\ell\right)
$$

where $a_{1, \ell}$ is the $\ell$ th component of $\boldsymbol{a}_{1}$. Note that the definition of $\mathbf{a}_{1}$ includes the received signal amplitude $\sqrt{\mathcal{E}}$ along with the channel gains $\beta_{1, \ell}$, but we continue to refer to it as the channel response for the sake of convenience. The estimated band-limited channel response can be defined analogously as

$$
\hat{h}_{c}(\xi)=\sum_{\ell=0}^{L_{c}-1} \hat{a}_{1, \ell} \operatorname{sinc}\left(W \xi-\hat{d}_{1}-\ell\right)
$$

where $\hat{a}_{1, \ell}$ is the $\ell$ th component of the estimate $\hat{\boldsymbol{a}}_{1}$.

Now, we would like to approximate this estimated response by an $L_{r}$ tap model, and we allow for arbitrary spacings between

\footnotetext{
${ }^{4}$ This could happen in cases where the signal is received from a small number of strong reflectors.
} 
the taps to improve the approximation. Consequently, the $L_{r}$ tap model can be written using (6) as

$$
h_{r}(\xi)=\sum_{m=1}^{L_{r}} \alpha_{m} \operatorname{sinc}\left(W \xi-\hat{d}_{1}-\tau_{m}\right)
$$

where $\alpha_{m}$ are the amplitudes in the new model and $\left\{\tau_{m} T_{c}\right\}$ are the delays relative to $\hat{d}_{1} T_{c}$. Essentially, $\left\{\tau_{m} T_{c}\right\}$ are (continuous) variables chosen in the range $\left[0, L_{c} T_{c}\right]$. However, to ensure that the taps in the reduced tap channel see approximately independent interference, we impose the restriction that no two taps are less than a chip apart, i.e., that $\left|\tau_{i}-\tau_{j}\right| \geq 1$ for $i \neq j$.

Clearly, we need to define the sense in which $h_{r}(\xi)$ approximates $\hat{h}_{c}(\xi)$. If we consider them to be functions in $\mathcal{L}^{2}[-\infty, \infty]$ with the inner product

$$
\langle f, g\rangle=\int_{-\infty}^{\infty} f(\xi) g^{\star}(\xi) d \xi
$$

the correlation coefficient can be defined as

$$
\rho(f, g)=\frac{\langle f, g\rangle}{\|f\|\|g\|}, \quad \text { with the norm }\|f\|=\sqrt{\langle f, f\rangle} .
$$

Now, if the Rake receiver (maximum ratio combiner) corresponding to the function $h_{r}$ is used, it is shown in the Appendix that the output SIR is proportional to $\left|\rho\left(h_{c}, h_{r}\right)\right|^{2}$. This motivates the use of the magnitude of the correlation coefficient between the estimated channel $\hat{h}_{c}(\xi)$ and $h_{r}(\xi)$ as the performance metric for the second stage, and we refer to it simply as the correlation. Note that the correlation of discrete channel vectors has been used as a performance metric elsewhere in the literature (e.g., [13]).

The problem then is to find $h_{r}(\xi)$ that maximizes the absolute value of the correlation, $\left|\rho\left(h_{c}, h_{r}\right)\right|$. In addition, we impose the constraint that the norm of $h_{r}(\xi)$ must be the same as that of $\hat{h}_{c}(\xi)$, so that the received signal energy does not change with the choice of the channel model. Thus,

$$
\begin{aligned}
h_{r}(\xi) & =\arg \max _{h_{r}:\left\|h_{r}\right\|=\left\|\hat{h}_{c}\right\|} \frac{\left|\left\langle\hat{h}_{c}, h_{r}\right\rangle\right|}{\left\|\hat{h}_{c}\right\|\left\|h_{r}\right\|} \\
& =\arg \max _{h_{r}:\left\|h_{r}\right\|=\left\|\hat{h}_{c}\right\|}\left|\left\langle\hat{h}_{c}, h_{r}\right\rangle\right| .
\end{aligned}
$$

The constraint thus reduces the problem to one of maximizing the absolute value of the inner product. Now, $\left\langle\hat{h}_{c}, h_{r}\right\rangle$ can be written in terms of the tap parameters as

$$
\begin{aligned}
\left\langle\hat{h}_{c}, h_{r}\right\rangle= & \sum_{\ell=0}^{L_{c}-1} \sum_{m=1}^{L_{r}} \alpha_{m}^{\star} \hat{a}_{1, \ell} \\
& \cdot\left\langle\operatorname{sinc}\left(W \xi-\hat{d}_{1}-\tau_{m}\right), \operatorname{sinc}\left(W \xi-\hat{d}_{1}-\ell\right)\right\rangle \\
= & \frac{1}{W} \sum_{\ell=0}^{L_{c}-1} \sum_{m=1}^{L_{r}} \alpha_{m}^{\star} \hat{a}_{1, \ell} \operatorname{sinc}\left(\tau_{m}-\ell\right) \\
= & \frac{1}{W} \boldsymbol{\alpha}^{\dagger} X \hat{\boldsymbol{a}}_{1}
\end{aligned}
$$

where $\boldsymbol{\alpha}=\left[\begin{array}{lll}\alpha_{1} & \ldots & \alpha_{L_{r}}\end{array}\right]^{\top}$ and the $(m, \ell+1)$ th component of $\boldsymbol{X}$ is given by $X_{m, \ell+1}=\operatorname{sinc}\left(\tau_{m}-\ell\right)$. Similarly,

$$
\left\|h_{r}\right\|^{2}=\left\langle h_{r}, h_{r}\right\rangle=\frac{1}{W} \boldsymbol{\alpha}^{\dagger} \boldsymbol{Y} \boldsymbol{\alpha}
$$

where $(i, j)$ th component of $\boldsymbol{Y}$ is $Y_{i j}=\operatorname{sinc}\left(\tau_{i}-\tau_{j}\right)$. Hence, defining $\boldsymbol{\tau}=\left[\begin{array}{lll}\tau_{1} & \ldots & \tau_{L_{r}}\end{array}\right]^{\top}$, the parameters for the reduced tap channel are given by

$$
[\hat{\boldsymbol{\tau}}, \hat{\boldsymbol{\alpha}}]=\arg \max _{\boldsymbol{\tau}, \boldsymbol{\alpha}: \boldsymbol{\alpha}^{\dagger} \boldsymbol{Y} \boldsymbol{\alpha}=\boldsymbol{a}_{1}^{\dagger} \hat{\boldsymbol{a}}_{1}}\left|\frac{1}{W} \boldsymbol{\alpha}^{\dagger} \boldsymbol{X} \hat{\boldsymbol{a}}_{1}\right|
$$

Using the Cauchy-Schwarz inequality, it is easy to show that the solution is

$$
\begin{aligned}
& \hat{\boldsymbol{\tau}}=\arg \max _{\boldsymbol{\tau}} \hat{\boldsymbol{a}}_{1}^{\dagger} \boldsymbol{X}(\boldsymbol{\tau})^{\dagger} \boldsymbol{Y}(\boldsymbol{\tau})^{-1} \boldsymbol{X}(\tau) \hat{\boldsymbol{a}}_{1} \\
& \hat{\boldsymbol{\alpha}}=\frac{\boldsymbol{Y}^{-1} \boldsymbol{X} \hat{\boldsymbol{a}}_{1}\left\|\hat{\boldsymbol{a}}_{1}\right\|}{\left\|\hat{\boldsymbol{a}}_{1}^{\dagger} \boldsymbol{X}^{\dagger} \boldsymbol{Y}^{-1} \boldsymbol{X} \hat{\boldsymbol{a}}_{1}\right\|}
\end{aligned}
$$

where the vector norms are the usual Euclidean norms.

While this approach maximizes the correlation between the $L_{r}$ tap model and estimated $L_{c}$ tap model, it is important to note that this correlation is not the performance measure for the algorithm over both stages. The overall metric is the correlation magnitude $\left|\rho\left(h_{c}, h_{r}\right)\right|$ of the reduced-tap channel with the actual (and, of course, unknown) channel, which would involve the inner product

$$
\begin{aligned}
\left\langle h_{c}, h_{r}\right\rangle & =\frac{1}{W} \sum_{\ell=0}^{L_{c}-1} \sum_{m=1}^{L_{r}} \alpha_{m}^{\star} a_{1, \ell} \operatorname{sinc}\left(\hat{d}_{1}+\tau_{m}-d_{1}-\ell\right) \\
& =\frac{1}{W} \boldsymbol{\alpha}^{\dagger} \boldsymbol{Z} \boldsymbol{a}_{1}
\end{aligned}
$$

where $Z_{m, \ell+1}=\operatorname{sinc}\left(\hat{d}_{1}+\tau_{m}-d_{1}-\ell\right)$. The MMSE-ML algorithm followed by the correlation-based tap reduction is thus not optimal in the overall sense. In addition, note that the solution (27) involves a joint maximization over $L_{r}$ delays (albeit over a restricted search space). Hence, we propose other simpler (but more $a d$ hoc) approaches below.

Alternative Approaches to Reducing the Number of Taps: The first approach is to simply pick the $L_{r}$ most significant taps of the $L_{c}$ taps in $\hat{h}_{c}(\xi)$. We refer to this as the "Max- $L_{r}$ " approach in next section.

We can improve on the "Max- $L_{r}$ " technique as follows. Consider again the MMSE-ML solution for the $L_{c}$ tap channel in (19) and (20). Although this solution was derived based on a single delay parameter $d_{1}$, it is easy to see that the solution can also be applied to an arbitrarily spaced channel model, where the matrix $\boldsymbol{U}$ is a function of the delay vector $\boldsymbol{\tau}$. In order to find the reduced tap channel, we could hence perform a brute force maximization of the metric in (20) over $\tau$. However, instead of performing the $L_{r}$ variable maximization over the entire window $\left[d_{1}, d_{1}+L_{c}-1\right]$, we restrict our search space to a \pm 1 neighborhood around each of the $L_{r}$ most significant taps of the $L_{c}$ taps in $\hat{h}_{c}(\xi)$. The reason for this restriction of the search space is based on the intuition that the $L_{r}$ taps which best approximate $\hat{h}_{c}(\xi)$ would be in the vicinity of the most significant taps in $\hat{h}_{c}(\xi)$.

We will see in the next section that these ad hoc approaches to tap reduction result in some performance loss with respect to the optimum correlation scheme. 


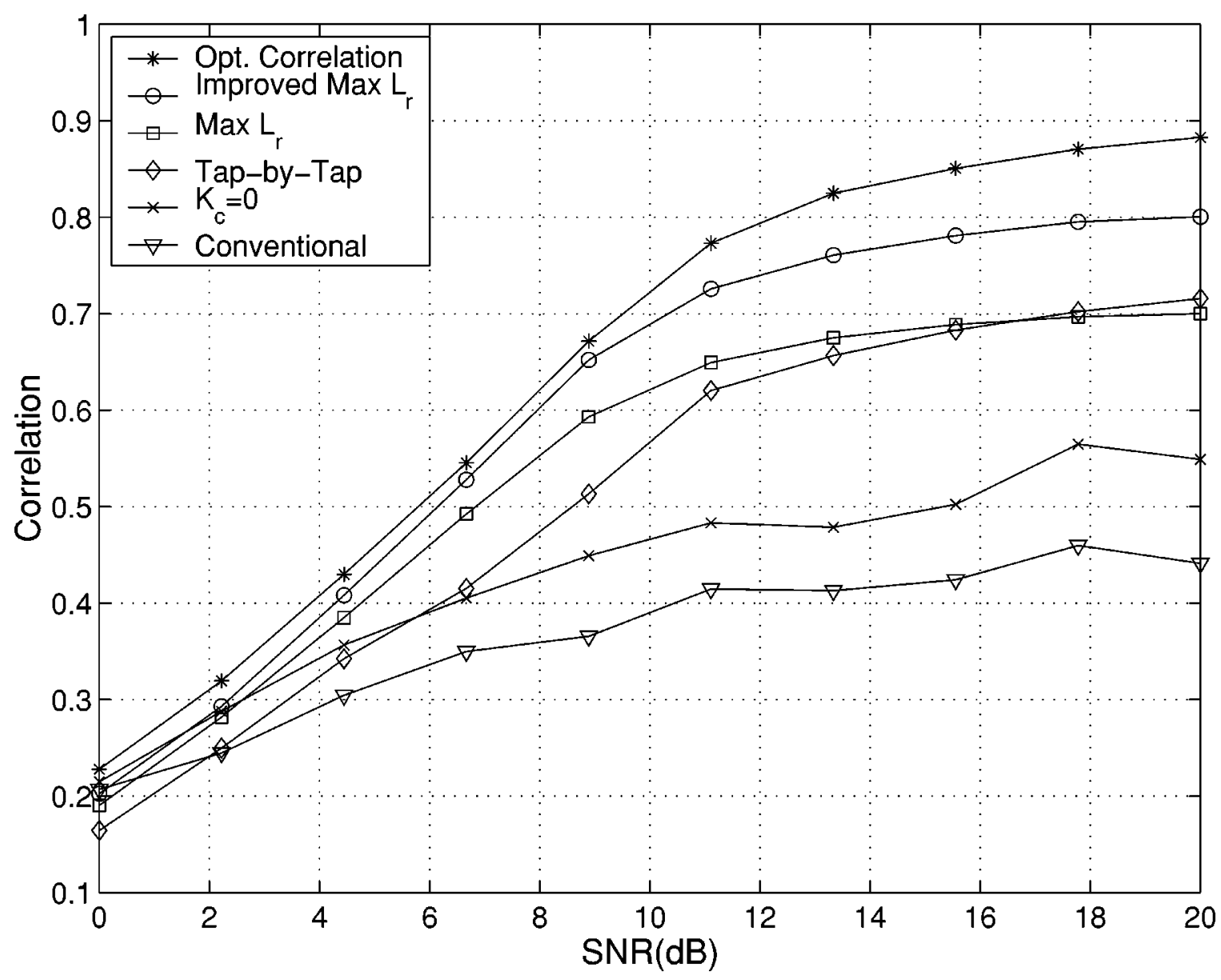

Fig. 1. Performance with variation in SNR, $\mathcal{E}_{1} / N_{o}$, with a deterministic equal gain channel for the desired user and independent Rayleigh fading channels with equal tap variances for the interferers, with $L_{c}=6, L_{r}=3, M=3, b=1$.

\section{NUMERICAL RESULTS}

We now study the performance of our acquisition scheme through simulations. Throughout the simulations, the spreading factor is fixed at $N=31$ and the delay uncertainty at $J=30$. The chip waveform is taken to be a sinc pulse truncated to a length of 9 chips (the truncation results in about $2 \%$ loss in energy of the pulse), and the chip sequences are assumed to take on binary $\pm 1 / \sqrt{N}$ values (BPSK spreading). In addition, we set the number of users in the system to $K=10$ and assume that the average received powers $\mathcal{E}_{k}$ from all the users are the same. Finally, the joint maximization over continuous delays required in the second stage of our proposed algorithm is approximated by using discrete delays with a resolution of 5 samples per chip.

In order to illustrate the improvement in performance that can be achieved by using an MAI resistant joint estimation scheme, we compare our schemes to three special cases in our general framework. We refer to these as the " $K_{c}=0$," "tap-by-tap," and "conventional" acquisition schemes. The " $K_{c}=0$ " scheme treats all the interfering users as random noise, but continues to estimate the channel coefficients jointly. This essentially amounts to setting $\boldsymbol{B}=\boldsymbol{I}$ in the estimators (19) and (20). Once the $L_{c}$ tapped channel estimate is obtained, the optimal correlation maximizing second stage is implemented.

The "tap-by-tap" acquisition scheme uses an implicit MMSE estimate of the symbols $\boldsymbol{b}$, but estimates the channel tap by tap instead of jointly. This scheme maximizes the single tap ML metric, which can be seen as a special case of (20) when we assume a single tap in the channel, i.e., $L_{c}=1$. The estimate of a particular tap is given by

$$
\begin{aligned}
\hat{\tau} & =\arg \max _{\tau_{1}} \frac{\left|\boldsymbol{u}_{1}\left(\tau_{1}\right)^{\dagger} \boldsymbol{B} \boldsymbol{y}\right|^{2}}{\boldsymbol{u}_{1}\left(\tau_{1}\right)^{\dagger} \boldsymbol{B} \boldsymbol{u}_{1}\left(\tau_{1}\right)} \\
\hat{a}_{1} & =\frac{\boldsymbol{u}_{1}\left(\hat{\tau}_{1}\right)^{\dagger} \boldsymbol{B} \boldsymbol{y}}{\boldsymbol{u}_{1}\left(\hat{\tau}_{1}\right)^{\dagger} \boldsymbol{B} \boldsymbol{u}_{1}\left(\hat{\tau}_{1}\right)} .
\end{aligned}
$$

The channel is again estimated in two stages. In the first stage, the single path ML metric is evaluated at the $J$ chip boundaries in the uncertainty region and the maximum is picked. The search space is then restricted to a $2 L_{c} T_{c}$ region about this position, the single tap metric is computed over the restricted space, and the delays corresponding to the $L_{r}$ maximum values are picked. The delay values are also restricted so that no two taps can be less than a chip apart, and so that the $L_{r}$ taps do not have a span that is greater than $\left\lceil\tau_{D S} W\right\rceil$.

Finally, the "conventional" scheme involves setting $K_{c}=0$ as well as estimating the channel tap by tap as described above, i.e., it involves neither MAI suppression nor joint acquisition of the taps.

The performance of the proposed acquisition schemes is illustrated by observing the magnitude of the correlation, $\left|\rho\left(h_{c}, h_{r}\right)\right|$. In all cases, the channel is taken to have $L_{c}=6$ 


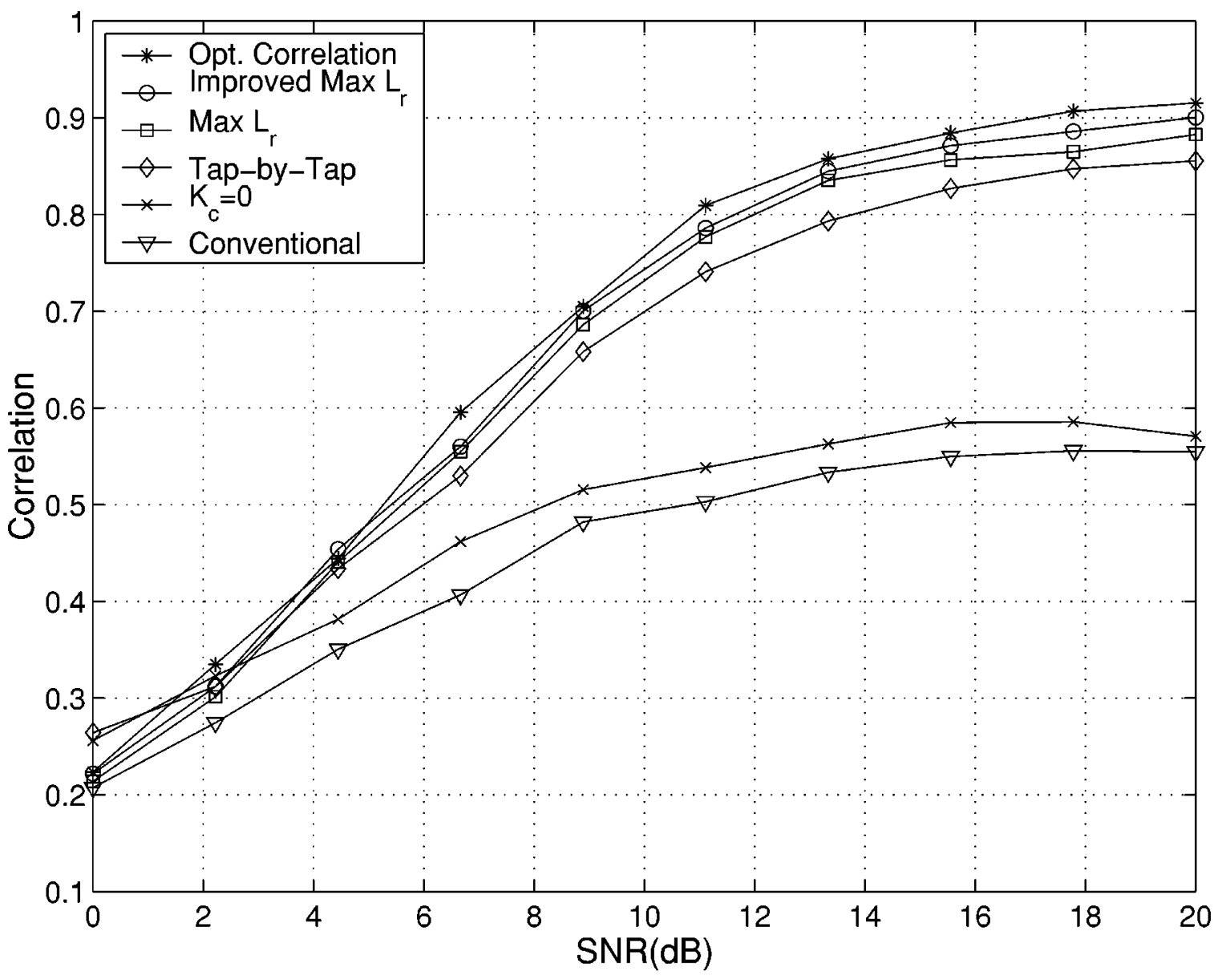

Fig. 2. Performance with variation in SNR, $\mathcal{E}_{1} / N_{o}$, with independent Rayleigh fading channels with equal tap variances for all users, with $L_{c}=6, L_{r}=$ $3, M=3, b=1$.

taps. For the interfering users, it is assumed that each tap undergoes independent Rayleigh fading, i.e., the channel gain coefficients $\beta_{k, \ell}$, for $k=2, \ldots, K$ and $\ell=1, \ldots, L_{c}$ are i.i.d. zero mean CCG random variables with equal variances, $E\left|\beta_{k, \ell}\right|^{2}=1 / L_{c}$. We also assume that the coherence time of the channel is longer than the preamble length; hence, the coefficients $\left\{\beta_{k, \ell}\right\}$ are constant over the observation interval and are generated independently across different simulation trials.

For the desired user, we consider two scenarios for the actual channel. In the first case, all $L_{c}$ taps $\beta_{1, \ell}$ are deterministic and equal to $1 / \sqrt{L_{c}}$. Although such a channel is rarely encountered in practice, it is interesting to study the performance of the proposed schemes in this special case. In the second case, we assume the same channel model for the desired user as that for the interfering users: i.i.d. Rayleigh fading with equal variances. Finally, we choose $L_{r}=3$ as the number of taps in the reduced channel.

The performance with equal deterministic $\beta_{1, \ell}$ is shown in Fig. 1. These performance curves are obtained when the preamble of the new user is of length $M=3$ symbols and processing is done once over the entire preamble. It is clear from these curves that significant gains can be obtained if the scheme exploits the structure of the interferers and estimates the channel coefficients jointly. The "opt. correlation" scheme in the second stage achieves the highest overall correlation and approaches an asymptotic value of approximately 0.9 , and both the second stage schemes perform better than the "max $L_{r}$ " scheme justifying the need for a more sophisticated second stage. Since the "tap-by-tap" scheme suppresses MAI, it does not suffer from a noise floor like the " $K_{c}=0$ " and "conventional" schemes. The "tap-by-tap" scheme is therefore able to capture most of the energy as long as it has estimated the strongest tap correctly, and this becomes more likely at high SNR's. In fact, this scheme could perform better than the "max $L_{r}$ " scheme at high SNR's, since it allows for arbitrary spacing of the taps.

It is interesting to note that the schemes that do not suppress MAI (namely, " $K_{c}=0$ " and "conventional") perform better than the remaining schemes at sufficiently low SNR's. This behavior arises because of the unreliability of the implicit estimate, $\hat{\boldsymbol{b}}$, of the interfering symbols at low SNR's. It is analogous to the cross-over behavior at low SNR observed in [7] for single path acquisition, and in [11] for the decorrelating detection problem.

Fig. 2 shows the same curves when $\beta_{1, \ell}$ are i.i.d. CCG random variables. The proposed schemes perform better in this environment as opposed to the previous one, since the tap gains would in general be unequal for each realization, and using a reduced channel with just three taps suffices to obtain a good approximation. The deterministic equal gain channel can thus be viewed as the worst-case situation for tap reduction, since 


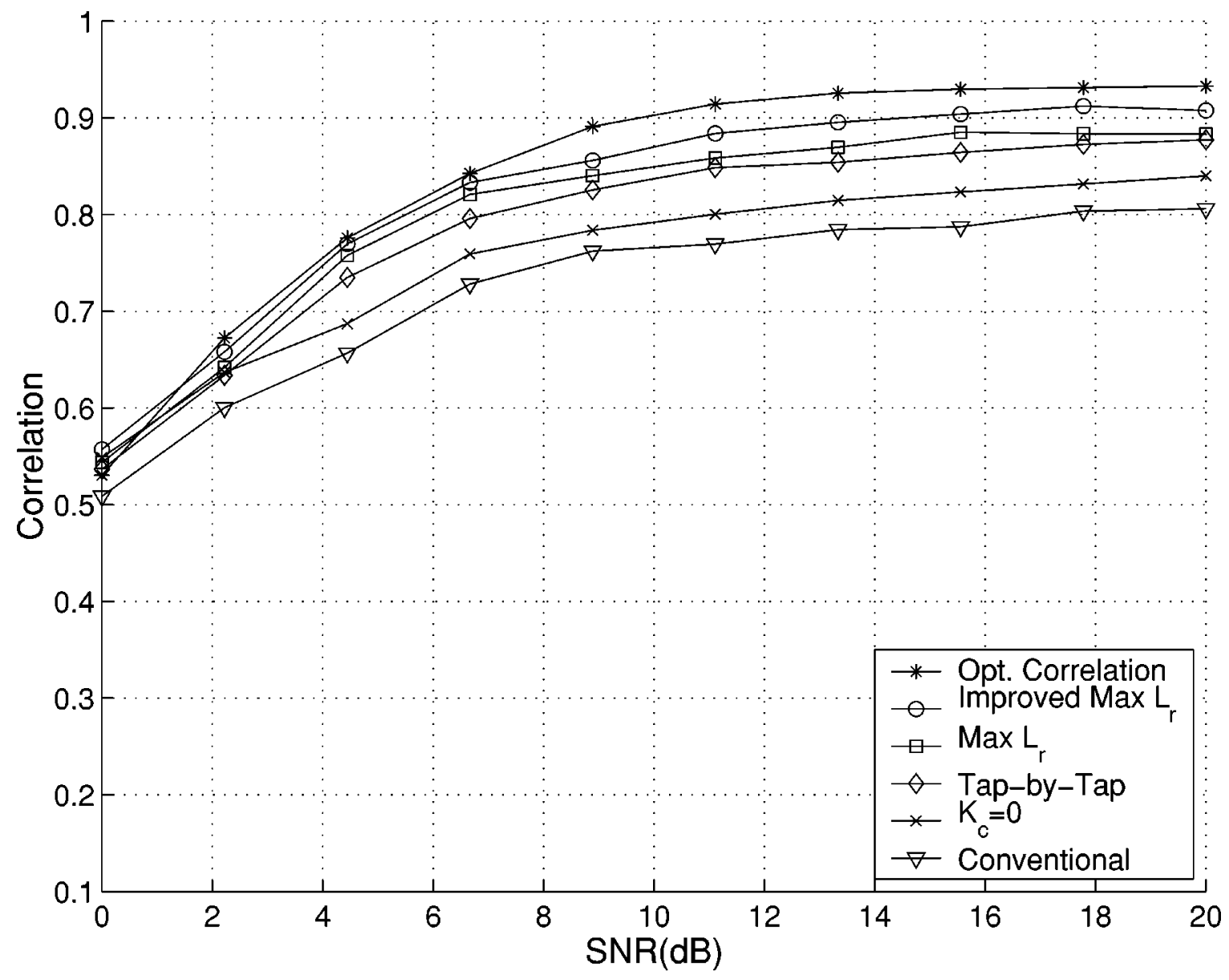

Fig. 3. Performance with variation in SNR, $\mathcal{E}_{1} / N_{o}$, with independent Rayleigh fading channels with equal tap variances for all users, with $L_{c}=6$, $L_{r}=$ $3, M=3, b=3$. This plot shows the improvement in performance that results from the use of multiple blocks for the estimation.

a significant fraction of the energy may not be captured. In addition, note that the performance gap between the different second stage schemes reduces when the gains are random. If the weaker taps for each realization are insignificant compared to the strongest $L_{r}$ taps, then fine tuning the tap positions becomes unnecessary. Hence, there may not be any need for a sophisticated second stage at high SNR's, as the "max $L_{r}$ " scheme may attain the same correlation as the optimum second stage.

Now, as discussed in Section III, the SNR required to achieve the same performance can be reduced for any of the schemes by processing multiple blocks using (21). This is shown in Fig. 3, where we have used $b=3$ blocks instead of one, with $M=3$ as before. Note that averaging over multiple blocks reduces the noise floor for the $K_{c}=0$ schemes and, hence, the performance of these schemes in Fig. 3 is significantly better than that in Fig. 2.

Finally, we study the loss in performance resulting due to tap reduction in Fig. 4. As before, we set $L_{c}=6$ with i.i.d. Rayleigh fading on each tap for the desired user. As SNR increases, the asymptotic value of the correlation is limited by $L_{r}$, and a sufficiently high value of $L_{r}$ should be chosen to obtain the desired performance. In Fig. 4, for instance, depending on system requirements, the minor improvement that $L_{r}=5$ taps provides over $L_{r}=4$ may not justify the additional complexity.

\section{CONCLUSION}

In this paper, we considered reverse link channel estimation algorithms for a single new user entering a wideband CDMA system that uses random spreading sequences. A maximum likelihood solution was obtained for the channel parameters after using implicit soft MMSE estimates for the symbols of the interfering users. The initial problem formulation involved a channel model with equally spaced taps. Schemes to reduce the number of taps by allowing for arbitrary spacing between them were also proposed. Numerical results presented indicate that MAI resistant joint tap acquisition schemes provide significant gains over schemes that do not exploit the structure of interfering users or those that acquire paths individually. Most of the computation in the scheme is taken up in forming the soft MMSE estimates and the $L_{r}$ variable optimization over the delays in the second stage of tap reduction. However, unlike multiuser detection schemes that involve updating the detector when the spreading sequences change, the initial channel estimation is not as severely time constrained, and it should be possible to allow for some complexity. Moreover, the proposed schemes allow for a tradeoff between performance and complexity through the parameters $L_{r}$, the final number of taps, and $K_{c}$, the number of interferers suppressed. In particular, for $K_{c}=0$ and $L_{r}=L_{c}=1$, the MMSE-ML metric reduces to the single tap scheme based on a matched-filter statistic that is used in IS-95 based systems [16]. 


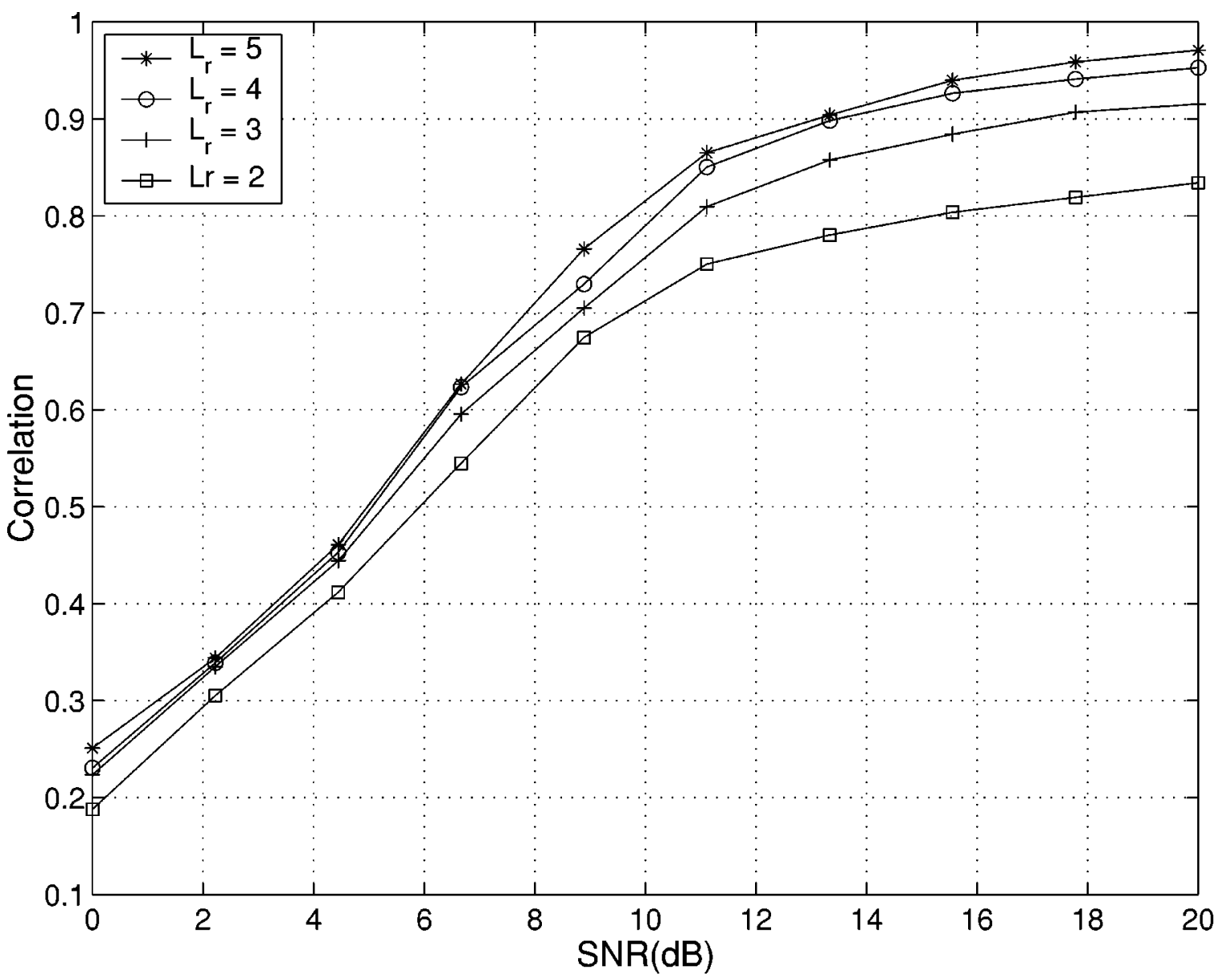

Fig. 4. Performance with variation in SNR, $\mathcal{E}_{1} / N_{o}$, with independent Rayleigh fading channels with equal tap variances for all users, with $L_{c}=6, M=3, b=$ 1 , with varying $L_{r}$.

A key assumption in arriving at the solution was that the channel gains and spreading sequences of the other users were known. If knowledge of the channel gains is not assumed, it is possible to take a decorrelation approach to suppress interference [7], but such a scheme would have a limitation on the number of interferers that can be suppressed, and the limitation would become more stringent as the number of resolvable paths increases. It is worth noting that the MMSE-ML scheme proposed in this paper imposes no constraint on the number of interferers in the system, but the robustness of the scheme to the knowledge of these channel parameters needs to be investigated further.

It is possible to incorporate any a priori information about the distribution of the channel gains of the new user into the MMSE-ML estimate, as done in [10]. However, the availability of such information at the receiver is open to question, and the resulting improvement needs to be investigated. Finally, proposals for third generation systems (e.g., [15]) provide the option of having users with different data rates. If the multiple rates were accommodated by using multiple spreading sequences with the same spreading factor, it is clear that the MMSE-ML solution can be applied with no modification. If the spreading factors vary across the users, it is still possible to apply the solution by identifying the effective users appropriately.

\section{APPENDIX}

We show that the output SNR of a Rake receiver that corresponds to the $L_{r}$ tap model, $h_{r}$, is approximately proportional to the square of correlation coefficient between $h_{r}$ and the actual $L_{c}$ tap model, $h_{c}$.

Suppose, the complex baseband signal $s(t)=b_{0} c_{1}(t)$ is transmitted through the $L_{c}$ tap channel, where $b_{0}$ is the transmitted symbol, and $c_{1}(t)$ is the symbol waveform. The received complex baseband signal can be expressed as

$$
r(t)=\sum_{\ell=0}^{L_{c}-1} a_{\ell} b_{0} c_{1}(t-\ell / W)+w(t), \quad t \in[0, T]
$$

where $a_{\ell}=\beta_{\ell} \sqrt{\mathcal{E}}, T$ is the observation interval and $w(t)$ is assumed to be a complex white random process with autocorrelation function $N_{0} \delta(\tau)$. Suppose the receiver assumes a channel model with $L_{r}$ taps and amplitudes $\alpha_{m}, m=1, \ldots L_{r}$. Then, the statistic of the Rake receiver is given by

$$
\begin{aligned}
r & =\sum_{m=1}^{L_{r}} \sum_{\ell=0}^{L_{c}-1} \alpha_{m}^{\star} a_{\ell} b_{0} \int_{0}^{T} c_{1}\left(t-\ell T_{c}\right) c_{1}^{\star}\left(t-\tau_{m} T_{c}\right) d t+w \\
& =\sum_{m=1}^{L_{r}} \sum_{\ell=0}^{L_{c}-1} \alpha_{m}^{\star} a_{\ell} b_{0} \Gamma\left(\ell T_{c}-\tau_{m} T_{c}\right)+w
\end{aligned}
$$


where

$$
w=\sum_{m=1}^{L_{r}} \alpha_{m}^{\star} \int_{0}^{T} w(t) c_{1}\left(t-\tau_{m} T_{c}\right) d t
$$

and

$$
\begin{aligned}
& \Gamma(\tau)= \int_{0}^{T} c_{1}(t+\tau) c_{1}^{\star}(t) d t \\
& \approx W \int_{0}^{T} \sum_{j=0}^{N-1} \sum_{k=0}^{N-1} c_{1, j} c_{1, k}^{\star} \operatorname{sinc}(W t-j-0.5) \\
& \cdot \operatorname{sinc}(W t+W \tau-k-0.5) d t \\
&= \sum_{j=0}^{N-1} \sum_{k=0}^{N-1} c_{1, j} c_{1, k}^{\star} \operatorname{sinc}(W \tau-k+j) \\
&= \sum_{r} \operatorname{sinc}(W \tau-r) \sum_{j} c_{1, j} c_{1, r+j}^{\star}, \\
& \quad \text { where } r=k-j .
\end{aligned}
$$

Under the assumption that the spreading sequences have good autocorrelation properties,

$$
\sum_{j} c_{1, j} c_{1, r+j}^{\star} \approx \begin{cases}0 & \text { if } r \neq 0 \\ 1 & \text { if } r=0\end{cases}
$$

Note that this assumption is used in establishing the (approximate) optimality of the Rake receiver [9, p. 800]. Hence, we can rewrite (32) as

$$
\Gamma(\tau) \approx \operatorname{sinc}(W \tau)
$$

The Rake receiver statistic then reduces to

$$
\begin{aligned}
r & \approx b_{0} \sum_{m=1}^{L_{r}} \sum_{\ell=0}^{L_{c}-1} \alpha_{m}^{\star} a_{\ell} \operatorname{sinc}\left(\ell-\tau_{m}\right)+w \\
& =b_{0} W\left\langle h_{c}, h_{r}\right\rangle+w
\end{aligned}
$$

where we have used (28). The SNR at the output of the rake receiver is then given by

$$
\mathrm{SNR}_{o} \approx W^{2} \frac{\left|\left\langle h_{c}, h_{r}\right\rangle\right|^{2}}{\sigma_{w}^{2}}
$$

where $\sigma_{w}^{2}$ is the variance of $w$ and can be written, using (31) and (26), as

$$
\sigma_{w}^{2}=N_{0} \sum_{m=1}^{L_{r}} \sum_{\ell=1}^{L_{r}} \alpha_{m}^{\star} \alpha_{\ell} \operatorname{sinc}\left(\tau_{\ell}-\tau_{m}\right)=N_{0} W\left\|h_{r}\right\|^{2} .
$$

Finally, noting that $\left\|h_{c}\right\|^{2}=(1 / W) \sum_{\ell=0}^{L_{c}-1}\left|a_{\ell}\right|^{2}=(\mathcal{E} / W)$, we have

$$
\mathrm{SNR}_{\circ} \approx \frac{\mathcal{E}}{N_{0}}\left(\frac{\left|\left\langle h_{c}, h_{r}\right\rangle\right|}{\left\|h_{c}\right\||| h_{r} \|}\right)^{2}=\frac{\mathcal{E}}{N_{0}}\left|\rho\left(h_{c}, h_{r}\right)\right|^{2}
$$

which is the required result.

\section{REFERENCES}

[1] R. Cameron and B. Woerner, "Synchronization of CDMA systems employing interference cancellation," in Proc. VTC 1996, pp. 178-182.

[2] S. M. Kay, Fundamentals of Statistical Signal Processing: Estimation Theory. Englewood Cliffs, NJ: Prentice-Hall, 1993.

[3] U. Madhow, "Blind adaptive interference suppression for near-far resistant acquisition and demodulation of DS-CDMA signals," IEEE Trans. Signal Processing, vol. 45, pp. 124-36, Jan. 1997.

[4] —, "Blind adaptive interference suppression for direct-sequence CDMA," Proc. IEE, vol. 86, no. 10, pp. 2049-2069, Oct. 1998.

[5] U. Madhow and M. L. Honig, "MMSE interference suppression for direct-sequence spread-spectrum CDMA," IEEE Trans. Commun., vol. 42, pp. 3178-3188, Dec. 1994

[6] A. Mantravadi and V. Veeravalli, "On discrete sufficient statistics for detection asynchronous band-limited CDMA systems," in Proc. 1999 CISS. Baltimore, MD, Mar. 1999.

[7] - "Multiple-access interference resistant acquisition for band-limited CDMA systems with random sequences," IEEE J. Select. Areas Commun., vol. 18, no. 7, July 2000.

[8] S. Parkvall, "On the performance difference between long and short spreading sequences in DS/CDMA systems," in Proc. GLOBECOM, Sydney, Australia, Nov. 1998.

[9] J. G. Proakis, Digital Communications. New York: McGraw-Hill, 1995.

[10] R. R. Rick and L. B. Milstein, "Optimal decision strategies for acquisition of spread spectrum signals in frequency-selective fading channels," IEEE Trans. Commun., vol. 46, pp. 686-694, May 1998.

[11] R. Lupas and S. Verdu, "Near-far resistance of multi-user detectors in asynchronous channels," IEEE Trans. Commun., vol. 38, pp. 496-508, Apr. 1990.

[12] S. Bensley and B. Aazhang, "Subspace-based channel estimation for CDMA system," IEEE Trans. Commun., vol. 44, pp. 1009-1020, Aug. 1996.

[13] C. Sengupta et al., "Maximum likelihood multipath channel parameter estimation in CDMA systems," in Proc. 1998 CISS, Princeton, NJ, Mar. 1998.

[14] E. G. Storm et al., "Propagation delay estimation in asynchronous DS-CDMA systems," IEEE Trans. Commun., vol. 44, pp. 84-93, Jan. 1996.

[15] TIA cdma2000, "Wideband cdmaOne radio transmission technology proposal," Int. Telecommun. Union, Radiocommun. Study Groups, June 2000.

[16] TIA/EIA/IS-95, "Mobile station-base station compatibility standard for dual-mode wideband spread spectrum cellular system," Telecommun. Industry Assoc., July 1993.

[17] V. V. Veeravalli, "The coding-spreading tradeoff in CDMA systems," in Proc. 37th Annu. Allerton Conf., Monticello, IL, Sept. 1999.

[18] S. Vembu and A. J. Viterbi, "Two different philosophies in CDMA-A comparison," in Proc. IEEE 46th Vehic. Technol. Conf., Atlanta, GA, April 1996, pp. 869-873.

[19] A. Viterbi, CDMA: Principles of Spread Spectrum Communication. Reading, MA: Addison-Wesley, 1995.

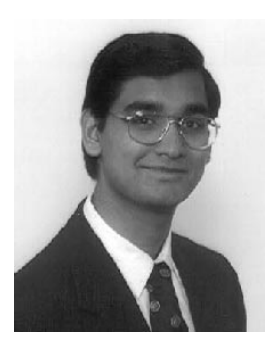

Vinayak Tripathi (S'00) was born on April 7, 1979. $\mathrm{He}$ received the B.S. (summa cum laude) degree in electrical engineering from Cornell University in 1997, and the M.S. degree in electrical engineering (systems) from the University of Michigan at Ann Arbor in 1998.

$\mathrm{He}$ is currently pursuing the $\mathrm{Ph} . \mathrm{D}$. degree at Cornell University. He received the Dean's Fellowship at the University of Michigan and the John McMullen Fellowship at Cornell University. His research interests include wireless communications, spread spectrum techniques, and packet data networks.

Mr. Tripathi is a member of Eta Kappa Nu and Tau Beta Pi. 


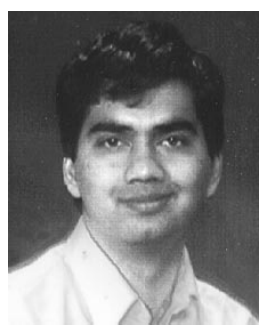

Ashok Mantravadi (S'97) received the B.Tech. degree in electronics and communications engineering from the Indian Institute of Technology, Madras, in 1996, and the M.S. degree from the School of Electrical Engineering, Cornell University, in January, in 1999.

Between June and August 1997, he worked as an Engineering Intern with the CDMA system design group at Qualcomm Inc., San Diego. He is currently in the Ph.D. program at Cornell University, where he has been a recipient of the John McMullen and Schlumberger Fellowships. His research interests include spread spectrum systems, wireless multiuser communications, and space-time signal processing.

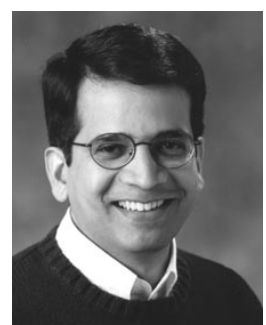

Venugopal V. Veeravalli (S'86-M'92-SM'98) received the Ph.D. degree in 1992 from the University of Illinois at Urbana-Champaign, the M.S. degree in 1987 from Carnegie-Mellon University, Pittsburgh, PA, and the B.Tech. degree in 1985 from the Indian Institute of Technology, Bombay (Silver Medal Honors), all in electrical engineering.

$\mathrm{He}$ joined the University of Illinois at $\mathrm{Ur}$ bana-Champaign in 2000, where he is currently an Associate Professor in the department of Electrical and Computer Engineering, and a Research Associate Professor in the Coordinated Science Laboratory. He was a postdoctoral fellow at Harvard University during 1992-1993, an Assistant Professor at the City College of NY during 1993-1994, a Visiting Assistant Professor at Rice University, Houston, TX during 1994-1996, and an Assistant Professor at Cornell University, Ithaca, NY during 1996-2000. His research interests include mobile and wireless communications, detection and estimation theory, and information theory.

Dr. Veeravalli is currently an Associate Editor for IEEE JSAC-Wireless Communication Series, and an Editor for Communications in Information and Systems (CIS). Among the awards he has received for research and teaching are the IEEE Browder J. Thompson Best Paper Award in 1996, the National Science Foundation CAREER Award in 1998, the Presidential Early Career Award for Scientists and Engineers (PECASE) in 1999, and the Michael Tien Excellence in Teaching Award from the College of Engineering, Cornell University in 1999. 